Journal of Mathematical Imaging and Vision, 4, 199-207 (1994).

(c) Kluwer Academic Publishers. Manufactured in The Netherlands.

\title{
FFT-Based Methods for Nonlinear Image Restoration in Confocal Microscopy
}

\author{
J.B.T.M. ROERDINK* \\ Centre for Mathematics and Computer Science, P.O. Box 4079, 1009 AB Amsterdam, The Netherlands
}

\begin{abstract}
Recently we developed a new method for attenuation correction in 3D imaging by a confocal scanning laser microscope (CSLM) in the (epi)fluorescence mode. The fundamental element in our approach consisted of multiplying the measured fluorescent intensity by a correction factor involving a convolution integral of this intensity, which can be computed efficiently by the fast Fourier transform (FFT). The resulting algorithm is one or two orders of magnitude faster than an existing iterative method, but it was found to have a somewhat smaller accuracy. In this paper we improve on this latter point by reformulating the problem as a statistical estimation problem. In particular, we derive first-order-moment and cumulant estimators leading to a nonlinear integral equation for the unknown fluorescent density, which is solved by an iterative method in which in each step a discrete convolution is performed by using the FFT. We find that only a few iterations are needed. It is shown that the estimators proposed here are more accurate than the existing iterative method, while they retain the advantage in computational efficiency of the FFT-based approach.
\end{abstract}

Key words. fluorescence confocal microscopy, attenuation correction, convolution method, fast Fourier transform, moment estimator, cumulant estimator, 3D image restoration, iterative methods

\section{Introduction}

A major problem in $3 \mathrm{D}$ imaging by a confocal scanning laser microscope (CSLM) in the (epi)fluorescence mode is the darkening of the deeper layers in the object due to scattering and absorption of excitation and fluorescence light [1], [7]. Visser et al. [6] have devised a method for correcting for this effect by layer stripping, in which one iteratively corrects the layers one at a time; see Visser et al. [6]. In previous research [5] we developed a new restoration method to correct for these effects. Assuming that the attenuation is weak, we constructed by analytic methods a correction factor to the standard restoration that takes the form of a $3 \mathrm{D}$ convolution of the measured signal, which can be efficiently computed by the use of the fast Fourier transform (FFT). We therefore refer to this method as the "FFT method." In this way, the complexity of computation is re-

\footnotetext{
*Present address: Dept. of Mathematics and Computing Science, University of Groningen, The Netherlands.
}

duced to $\mathcal{O}\left(N_{z} \log N_{z}\right)$, where $N_{z}$ is the number of vertical layers to be restored. The accuracy of the results depends on the depth of the layer considered; deeper layers are less accurately reconstructed than are shallower layers.

We also compared the computational efficiency of our algorithm with the iterative layerstripping method of [6], henceforth referred to as the "layer method." In its original form this method has computational complexity $\mathcal{O}\left(N_{z}^{4}\right)$, which is unacceptably slow, taking many hours on a RISC workstation for a $256 \times 256 \times 16$ image [6]. The layer method "with condensation," developed in [6] to reduce the computation time, still has complexity $\mathcal{O}\left(N_{z}^{2}\right)$. Thus when the number of vertical layers gets larger, the difference in computational efficiency between this method and the FFT method becomes increasingly pronounced. For spatially varying image densities the restoration quality of our method was found to be a little poorer than that of the layer method.

In this paper we improve the accuracy of the 
FFT method by reformulating the problem as a statistical estimation problem. In particular, we derive first-order-moment and cumulant estimators leading to a nonlinear integral equation for the unknown fluorescent density, which is solved by an iterative method. It is shown that the new estimators, the moment estimator in particular, are more accurate than the layer method. Since only two or three iterations are needed and each iteration step involves a discrete $3 \mathrm{D}$ convolution computable by the FFT, the advantage in computational efficiency over the layer method is retained.

The organization of this paper is as follows. In section 2 we review the mathematical model of the imaging process of the CSLM, leading to a nonlinear integral transform of the object function, and we review the solution method of [5]. In section 3 we then reformulate the CSLM transform as a statistical averaging problem and derive the corresponding first-order moment and cumulant estimators. The resulting nonlinear integral equations for the object density can be solved by an iterative method, which is described in section 4 . We apply our method in section 5 to the test images used in [5], and we present results on the restoration accuracy. Section 6 contains a summary and conclusions.

\section{The CSLM Transform}

The imaging process of a CSLM operating in the fluorescence mode was described in detail in [5]. A laser beam is focused on a pinhole, expanded again, and, through a system of lenses, focused on a point $\mathbf{r}=(x, y, z)$ in the object. Here the $z$ direction is chosen along the optical axis. The rays converging to the object point are contained in a circular cone (light cone) with angle $\omega$, called the "semiaperture angle"; see figure 1. The radiation absorbed at the point in focus is uniformly reemitted as fluorescent radiation, and the part that travels back the same route as the incoming radiation is detected. The object is discretized into a number $N_{z}$ of layers along the optical axis, a distance $\delta_{z}$ apart. The total depth of the sample is denoted by $d_{z}$. Also, each layer is discretized into a rectangular grid of $N_{x}$ by

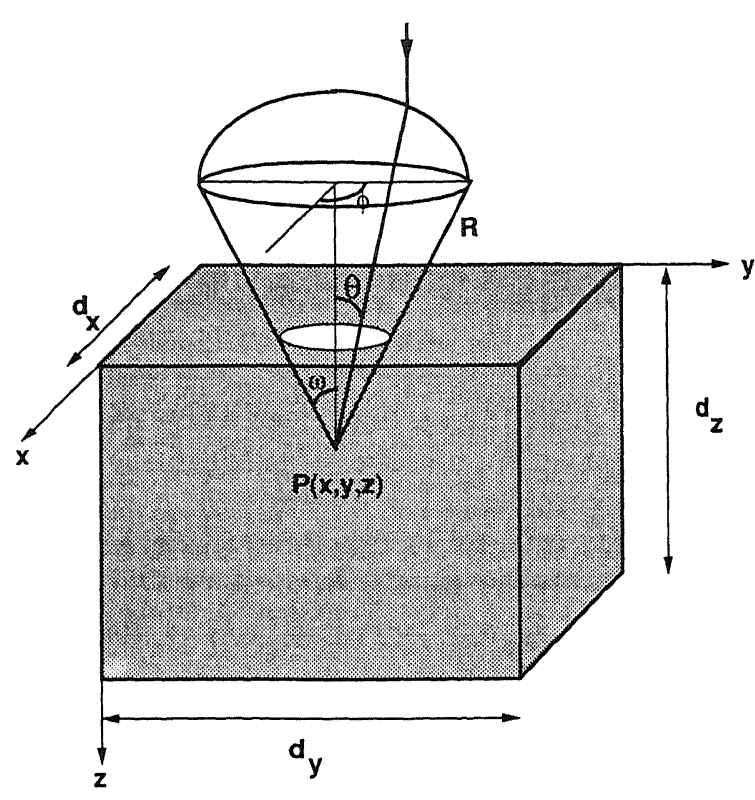

Fig. 1. Geometry of light cone with apex at a point $P(x, y, z)$ in the object: $R$, radius of spherical bundle; $\omega$, semiaperture angle; $(\theta, \phi)$, polar angles of light ray; $d_{z}$, depth of the sample. The optical axis coincides with the $z$ axis.

$N_{y}$ points, with spacings $\delta_{x}$ and $\delta_{y}$ in the $x$ and $y$ directions, respectively. When the scan table of the CSLM is moved, each object point of the 3D grid so formed is brought into focus and the corresponding fluorescent intensity (energy per unit of time) is measured.

As a result, the measured fluorescent intensity $f(\mathbf{r})$ can be expressed as the following nonlinear integral transform (CSLM transform) of the unknown fluorescent density $\rho(\mathbf{r})$ :

$$
f(\mathbf{r})=\rho(\mathbf{r}) \times \gamma_{f}(\mathbf{r}) \gamma_{b}(\mathbf{r}),
$$

where

$$
\begin{aligned}
\gamma_{f}(\mathbf{r}):= & C_{f} \int_{0}^{\omega} d \theta \int_{0}^{2 \pi} d \phi \sin \theta \cos \theta \\
& \times \exp \left[-\epsilon \int_{0}^{z} \frac{d z^{\prime}}{\cos \theta} \rho(\widehat{\mathbf{r}})\right]
\end{aligned}
$$

is the forward attenuation factor and

$$
\begin{aligned}
\gamma_{b}(\mathbf{r}):= & C_{b} \int_{0}^{\omega} d \theta \int_{0}^{2 \pi} d \phi \sin \theta \\
& \times \exp \left[-\epsilon \int_{0}^{z} \frac{d z^{\prime}}{\cos \theta} \rho(\widehat{\mathbf{r}})\right]
\end{aligned}
$$


is the backward attenuation factor (both factors equal unity if there is no attenuation). In these equations $\epsilon$ is a proportionality constant and

$$
C_{f}:=\frac{1}{\pi \sin ^{2} \omega}, \quad C_{b}:=\frac{1}{2 \pi(1-\cos \omega)}
$$

are the normalization constants referring to the forward and backward attenuation factors, respectively. Here $\widehat{\mathbf{r}}$ is the vector

$$
\begin{aligned}
\widehat{\mathbf{r}}\left(\mathbf{r} ; \theta, \phi, z^{\prime}\right)= & \left(x+\left(z-z^{\prime}\right) \tan \theta \cos \phi,\right. \\
& \left.y+\left(z-z^{\prime}\right) \tan \theta \sin \phi, z^{\prime}\right) .
\end{aligned}
$$

As $z^{\prime}$ runs from 0 to $z$ this vector describes a light ray traveling to the point $\mathbf{r}=(x, y, z)$ and making polar angles $\theta$ and $\phi$ with respect to the optical axis; see figure 1.

A measure for the degree of attenuation of the signal when it traverses the complete sample is provided by the dimensionless parameter $\epsilon d_{z}$, where $d_{z}$ is the depth of the sample. Here we have restricted ourselves to the case in which the attenuation is proportional to the fluorescent density. For a more general case see [5].

\subsection{Inversion of the CSLM Transform}

By performing a perturbation expansion of the density $\rho$ in the parameter $\epsilon$ and collecting terms to first order in $\epsilon$, we derived in [5] the following approximation $\tilde{\rho}(\mathbf{r})$ for the density:

$$
\widetilde{\rho}(\mathbf{r})=f(\mathbf{r})\{1+\epsilon c(\mathbf{r})\},
$$

where $c(\mathbf{r})$ is the convolution integral

$$
c(\mathbf{r})=\int_{-\infty}^{\infty} \int_{-\infty}^{\infty} \int_{-\infty}^{\infty} d \mathbf{r}^{\prime} \kappa\left(\mathbf{r}^{\prime}\right) f\left(\mathbf{r}-\mathbf{r}^{\prime}\right),
$$

with $\kappa(\mathbf{r})$, the space-invariant kernel, given by

$$
\begin{aligned}
& \kappa(x, y, z) \\
& =\left\{\begin{array}{cl}
C_{f} \frac{z}{\left(x^{2}+y^{2}+z^{2}\right)^{3 / 2}}+C_{b} \frac{1}{x^{2}+y^{2}+z^{2}}, \\
0 \leq z \leq d_{z}, \quad x^{2}+y^{2} \leq(z \tan \omega)^{2}, \\
0 \text { elsewhere, }
\end{array}\right.
\end{aligned}
$$

where it should be kept in mind that the densities $f$ and $\rho$ are nonzero only for $0 \leq z \leq d_{z}$. This means that in the computation of $c(x, y, z)$ only the part of the kernel between 0 and $z$ has to be taken into account; see figure 1 .

\subsection{Numerical Computation}

For numerical computation the integral (2.7) is discretized on a grid of $N_{x} \times N_{y} \times N_{z}$ voxels, each voxel being a box of dimensions $\delta_{x}, \delta_{y}, \delta_{z}$ in the $x, y$, and $z$ directions. Then the approximation (2.6) is replaced by

$$
R_{i j k}=F_{i j k}\left(1+\epsilon C_{i j k}\right)
$$

where

$$
\begin{aligned}
C_{i j k}= & \sum_{\substack{i^{\prime}=-M_{x} / 2+1 \\
M_{x} / 2}} \sum_{j^{\prime}=-M_{y} / 2+1}^{M_{y} / 2} \sum_{k^{\prime}=1}^{M_{z}} \\
& \times K_{i^{\prime} j^{\prime} k^{\prime}} F_{i-i^{\prime}, j-j^{\prime}, k-k^{\prime}},
\end{aligned}
$$

with

$$
\begin{aligned}
& R_{i j k}:=\widetilde{\rho}\left(i \delta_{x}, j \delta_{y}, k \delta_{z}\right), \\
& C_{i j k}:=c\left(i \delta_{x}, j \delta_{y}, k \delta_{z}\right), \\
& F_{i j k}:=f\left(i \delta_{x}, j \delta_{y}, k \delta_{z}\right)
\end{aligned}
$$

for $(i, j, k)$ in the index set $\mathbb{I}:=\{(i, j, k): i=$ $\left.1, \ldots, N_{x} ; j=1, \ldots, N_{y} ; k=1, \ldots, N_{z}\right\}$ and where it is tacitly understood that array elements are defined to be zero when the indices are not in the index set $\mathbb{I}$ (so the third summation in (2.10) actually runs from 1 to $k-1$ ). Here $K$ is the discrete counterpart of the convolution kernel (2.8),

$$
\begin{aligned}
K_{i j k}:= & \int_{(i-1 / 2) \delta_{x}}^{(i+1 / 2) \delta_{x}} d x \int_{(j-1 / 2) \delta_{y}}^{(j+1 / 2) \delta_{y}} d y \\
& \times \int_{(k-1 / 2) \delta_{z}}^{(k+1 / 2) \delta_{z}} d z \kappa(x, y, z) \\
\approx & \delta_{x} \delta_{y} \delta_{z} \kappa\left(i \delta_{x}, j \delta_{y}, k \delta_{z}\right),
\end{aligned}
$$

and $M_{x}=2 d_{z} \tan (\omega) / \delta_{x}, \quad M_{y}=2 d_{z} \tan (\omega) / \delta_{y}$, and $M_{z}=d_{z} / \delta_{z}=N_{z}$ denote the support of the kernel in the three space directions, where in all cases rounding to integer values is understood. Treating the $x$ and $y$ summations differently from 
the $z$ summation is justified because the kernel is symmetric in the $x$ and $y$ directions, whereas it extends over only nonnegative values in the $z$ direction. The discrete convolution (2.10) can be computed efficiently by FFT methods [5]; see also [4].

\section{Statistical Estimators}

It is useful to provide a probabilistic formulation of the CSLM transform. To this end we notice that by introducing the probability densities into $(\theta, \phi)$ space $\{(\theta, \phi): 0 \leq \theta \leq \omega, 0 \leq \phi<2 \pi\}$,

$$
\begin{aligned}
& p_{f}(\theta, \phi)=\frac{\sin \theta \cos \theta}{\pi \sin ^{2} \omega}, \\
& p_{b}(\theta, \phi)=\frac{\sin \theta}{2 \pi(1-\cos \omega)},
\end{aligned}
$$

we can rewrite the basic transform (2.1) as

$$
\begin{aligned}
f(\mathbf{r})= & \rho(\mathbf{r}) \mathbb{E}_{f}\left(\exp \left[-\epsilon \int_{0}^{z} \frac{d z^{\prime}}{\cos \theta} \rho(\hat{\mathbf{r}})\right]\right) \\
& \times \mathbb{E}_{b}\left(\exp \left[-\epsilon \int_{0}^{z} \frac{d z^{\prime}}{\cos \theta} \rho(\hat{\mathbf{r}})\right]\right)
\end{aligned}
$$

where $\mathbb{E}_{i}$ denotes the mathematical expectation (statistical average) with respect to the density $p_{i}, i=f, b$.

Now we can apply moment and cumulant expansions of characteristic functions [2]. Performing the first-order moment expansion for the random variable $X(\theta, \phi)$,

$$
\mathbb{E}_{i}(\exp [-\epsilon X(\theta, \phi)])=1-\epsilon \mathbb{E}_{i}(X(\theta, \phi))+\cdots,
$$

for both the forward and backward averages, we obtain

$$
\begin{aligned}
& f(\mathbf{r}) \simeq \rho(\mathbf{r})\left\{1-\epsilon \int_{0}^{2 \pi} d \phi \int_{0}^{\omega} d \theta C_{f} \sin \theta\right. \\
&\left.\times \int_{0}^{z} d z^{\prime} \rho\left(\widehat{\mathbf{r}}\left(\mathbf{r} ; \theta, \phi, z^{\prime}\right)\right)\right\} \\
& \times\left\{1-\epsilon \int_{0}^{2 \pi} d \phi \int_{0}^{\omega} d \theta C_{b} \tan \theta\right. \\
&\left.\times \int_{0}^{z} d z^{\prime} \rho\left(\hat{\mathbf{r}}\left(\mathbf{r} ; \theta, \pi, z^{\prime}\right)\right)\right\}
\end{aligned}
$$

Neglecting terms of order $\epsilon^{2}$ and rewriting the sum of the two first-order terms in convolution form as in [5, section 3], we obtain an equation for the moment approximation $\rho^{(m)}(\mathbf{r})$ of $(2.6)$,

$$
f(\mathbf{r})=\rho^{(m)}(\mathbf{r})\left\{1-\epsilon\left(\kappa * \rho^{(m)}\right)(\mathbf{r})\right\}
$$

where $\kappa * \rho$ denotes the convolution of the functions $\kappa$ and $\rho$ and the kernel $\kappa$ is identical to that in (2.8).

Next we look also at the first-order cumulant expansion,

$$
\begin{aligned}
& \mathbb{E}_{i}(\exp [-\epsilon X(\theta, \phi)]) \\
& \quad=\exp \left[-\epsilon \mathbb{E}_{i}(X(\theta, \phi))+\cdots\right]
\end{aligned}
$$

for both averages. Then we find the cumulant approximation $\rho^{(c)}(\mathbf{r})$ for the density, satisfying the equation

$$
f(\mathbf{r})=\rho^{(c)}(\mathbf{r}) \exp \left[-\epsilon\left(\kappa * \rho^{(c)}\right)(\mathbf{r})\right],
$$

where again the same kernel $\kappa$ turns up. To solve (3.5) and (3.7) numerically, we rewrite them as

$$
\rho^{(c)}(\mathbf{r})=f(\mathbf{r}) \exp \left[\epsilon\left(\kappa * \rho^{(c)}\right)(\mathbf{r})\right]
$$

for the cumulant estimator and as

$$
\rho^{(m)}(\mathbf{r})=f(\mathbf{r})\left[1-\epsilon\left(\kappa * \rho^{(m)}\right)(\mathbf{r})\right]^{-1}
$$

for the moment estimator. We assume that $\epsilon$ is chosen small enough for the inverse in (3.9) to exist. A precise condition can be derived by rewriting the convolution in this equation in the form (see [5, section 3])

$$
\begin{aligned}
\left(\kappa * \rho^{(m)}\right)(\mathbf{r})= & \int_{0}^{2 \pi} \int_{0}^{\omega} d \theta\left(C_{f} \sin \theta+C_{b} \tan \theta\right) \\
& \times \int_{0}^{z} d z^{\prime} \rho^{(m)}\left(\hat{\mathbf{r}}\left(\mathbf{r} ; \theta, \phi, z^{\prime}\right)\right),
\end{aligned}
$$

which yields

$$
\left|\left(\kappa * \rho^{(m)}\right)(\mathbf{r})\right| \leq 2 d_{z} \rho_{\max },
$$

where $d_{z}$ is the depth of the sample and $\rho_{\max }$ is the maximum value of the density $\rho$. So the preceding inverse exists if $\epsilon \leq\left(2 d_{z} \rho_{\max }\right)^{-1}$. 
If in (3.8) and (3.9) only first-order terms in $\epsilon$ are taken into account and $\rho^{(c)}$ or $\rho^{(m)}$ is replaced by $f$ in the right-hand sides of these equations, respectively, we recover approximation (2.6). It is therefore to be expected that the moment and cumulant estimators may give accurate results for a larger range of values of $\epsilon$ than the estimator used in [5]. This will be investigated further in section 5 .

\section{Computation by Iterative Algorithms}

After discretization of (3.8) and (3.9), a finite system of nonlinear equations of the form

$$
\begin{gathered}
R_{i j k}=F_{i j k} G\left((K * R)_{i j k}\right), \\
(i, j, k) \in \mathbf{I}
\end{gathered}
$$

results, where $G(x)=\exp (\epsilon x)$ and $G(x)=(1-$ $\epsilon x)^{-1}$, respectively, and $K * R$ is the discrete convolution of the $3 \mathrm{D}$ arrays $K$ and $R$. The following result is immediate.

LEMMA 4.1. Equations (4.1) have a unique solution.

Proof. We consider successive values of the depth variable $k$. Starting at $k=1$, we have

$$
R_{i j 1}=F_{i j 1} G\left((K * R)_{i j 1}\right) .
$$

Now, suppressing the summation limits in the $i$ and $j$ directions we have

$$
(K * R)_{i j 1}=\sum_{i^{\prime}, j^{\prime}} \sum_{k^{\prime}=1}^{M_{z}} K_{i^{\prime} j^{\prime} k^{\prime}} R_{i-i^{\prime}, j-j^{\prime}, 1-k^{\prime}}
$$

which equals zero because $R_{i j k}=0$ for $k \leq 0$. Since $G(0)=1$ for both estimators, we get

$$
R_{i j 1}=F_{i j 1} \text {. }
$$

Next, observe that for $k \geq 2$ we have

$$
(K * R)_{i j k}=\sum_{i^{\prime}, j^{\prime}} \sum_{k^{\prime}=1}^{k-1} K_{i^{\prime} j^{\prime} k^{\prime}} R_{i-i^{\prime}, j-j^{\prime}, k-k^{\prime}}
$$

so that there are only nonzero contributions from the previous $k-1$ vertical layers to the convolution. This means that (4.1) can be solved successively for layers $1,2, \ldots, N_{z}$, e.g.,

$$
R_{i j 2}=F_{i j 2} G\left(\sum_{i^{\prime}, j^{\prime}} K_{i^{\prime} j^{\prime} 1} F_{i-i^{\prime}, j-j^{\prime}, 1}\right),
$$

where we have used the previous result (4.2). The same argument shows that for any $n, R_{i j n}$ can be uniquely expressed in terms of the values of the previous $n-1$ layers of the signal array $F$. This completes the proof.

Equations (4.1) can be solved by Picard iteration [3], with $F_{i j k}$ as the initial estimate:

$$
\begin{aligned}
& R_{i j k}^{(1)}=F_{i j k} \\
& R_{i j k}^{(n)}=F_{i j k} G\left(\left(K * R^{(n-1)}\right)_{i j k}\right), \quad n=2,3, \ldots
\end{aligned}
$$

Each iteration step involves the computation of the discrete convolution $K * R^{(n-1)}$ of the estimate $R^{(n-1)}$ of the previous iteration (with the same convolution kernel $K$ ), which can be efficiently computed by the FFT. The first iterate of (4.5) with $G(x)=1+\epsilon x$ coincides with the discrete analogon of (2.6) and is the approximation used in [5].

The question of convergence of the iteration (4.5) is answered by the following proposition.

PROPOSITION 4.2. The iterates $R_{i j k}^{(n)}$ of (4.5) converge in a finite number of $N_{z}$ steps towards the unique solution of (4.1). The convergence is monotonic; that is, $R_{i j k}^{(n)} \geq R_{i j k}^{(n-1)}$.

Proof. We will prove the following assertion: after $n$ iterations, the array elements $R_{i j k}^{(n)}$ in layers 1 to $n$ have the correct values; that is, they coincide with the solution of (4.1). We use induction on $n$. The main ingredient is again (4.3), which for $k=1$ must be read as $(K * R)_{i j 1}=0$. The initial estimate

$$
R_{i j k}^{(1)}=F_{i j k}
$$

is correct for $k=1$; see (4.2). Next, assume that $R_{i j k}^{(n-1)}$ is correct for $k=1,2, \ldots, n-1$. Then, since $\left(K * R^{(n-1)}\right)_{i j k}$ involves only layers $1,2, \ldots, k-1$ of the array $R^{(n-1)}$, we can write, 
for $k=1,2, \ldots, n$,

$$
\begin{aligned}
R_{i j k}^{(n)} & =F_{i j k} G\left(\left(K * R^{(n-1)}\right)_{i j k}\right) \\
& =F_{i j k} G\left((K * R)_{i j k}\right) \\
& =R_{i j k} .
\end{aligned}
$$

So the induction hypothesis $R_{i j k}^{(n-1)}=R_{i j k}$ for $k=1,2, \ldots, n-1$ yields $R_{i j k}^{(n)}=R_{i j k}$ for $k=$ $1,2, \ldots, n$. This proves the assertion.

Finally, monotonicity of the iterates is easily proved by induction as well. First, because $G$ is increasing,

$$
R_{i j k}^{(2)}=F_{i j k} G\left(\left(K * R^{(1)}\right)_{i j k}\right) \geq F_{i j k}=R_{i j k}^{(1)} .
$$

Next, we assume that $R_{i j k}^{(n)} \geq R_{i j k}^{(n-1)}$ (induction hypothesis). Then, using (i) nonnegativity of the convolution kernel, (ii) the fact that $G$ is increasing, and (iii) the induction hypothesis, we deduce

$$
\begin{aligned}
R_{i j k}^{(n+1)} & =F_{i j k} G\left(\left(K * R^{(n)}\right)_{i j k}\right) \\
& \geq F_{i j k} G\left(\left(K * R^{(n-1)}\right)_{i j k}\right) \\
& =R_{i j k}^{(n)},
\end{aligned}
$$

and we are done.

In section 5 we apply the iterative procedure of this proposition for improving the image restorations as described in [5]. We will see that only a few iterations are needed for obtaining accurate results. If this were not the case and the full $N_{z}$ iterations were needed, then the complexity of our algorithm would increase from $\mathcal{O}\left(N_{z} \log N_{z}\right)$ (single convolution) to $\mathcal{O}\left(N_{z}^{2} \log N_{z}\right)$ and the advantage of our method over the layer method with condensation of Visser et al. [6], which has complexity $\mathcal{O}\left(N_{z}^{2}\right)$, would be lost.

A final result concerns the relative ordering of the two estimators considered.

Proposition 4.3. The estimators $\rho^{(m)}$ and $\rho^{(c)}$ satisfy the inequality

$$
\rho^{(c)} \leq \rho^{(m)} \text {. }
$$

This can be proved by complete induction, just as in the proof of Proposition 4.2. It seems very hard to obtain any general statement as to the ordering of these estimators with respect to the exact solution $\rho$. When the exact density $\rho$ depends only on $z$, one may show by convexity arguments that

$$
\rho^{(c)} \leq \rho \leq \rho^{(m)}
$$

pointwise (that is, for every $z$ ). In that case it is also clear that the cumulant estimator is more accurate than the approximation (2.6). For the following examples with densities varying in the $x$ and $y$ directions these inequalities are found to be satisfied as well.

\section{Restoration of a Test Image}

In this section we consider a test density (trigonometric image) $\rho(\mathbf{r})$ with a sinusoidal spatial variation which was used in [5]:

$$
\rho(\mathbf{r})=\frac{1}{4} \cos \left(2 \pi n_{x} x / d_{x}\right) \cos \left(2 \pi n_{y} y / d_{y}\right),
$$

where $d_{x}$ and $d_{y}$ are the spatial dimensions of the sample in the $x$ and $y$ directions, respectively. Signal data $F_{i j k}$ were generated by numerically computing the integrals in (2.1) for a number of equidistant $3 \mathrm{D}$ positions. The parameters were chosen as follows: $d_{x}=d_{y}=1.0 ; d_{z}=$ $0.1 ; N_{x}=N_{y}=128 ; N_{z}=8 ; \omega=1.04719 ; n_{x}=$ $n_{y}=8$. We computed the relative root-meansquare error,

$$
\mathcal{E}(z):=\left(\frac{\sum_{x=1}^{N_{x}} \sum_{y=1}^{N_{y}}\{\rho(x, y, z)-\widetilde{\rho}(x, y, z)\}^{2}}{\sum_{x=1}^{N_{x}} \sum_{y=1}^{N_{\nu}}\{\rho(x, y, z)\}^{2}}\right)^{1 / 2},
$$

between the original density $\rho$ and the restored density $\widetilde{\rho}$ at each plane $z=$ constant. Computations were performed on a SPARC workstation (35 MHz, $26 \mathrm{MIPS}$ ), taking about $1 \mathrm{~min}$ per iteration step (see [5, table 2]).

Table 1 shows results for the moment estimator (3.9), and table 2 shows results for the cumulant estimator (3.8). For the moment estimator the errors first decrease and then start to 
Table 1. Signal error and restoration errors by the moment estimator $\rho^{(m)}$, after one, two, and three iterations, as a function of the effective depth $\epsilon z$.

\begin{tabular}{lllll}
\hline$\epsilon z$ & signal & iter $=1$ & iter $=2$ & iter $=3$ \\
\hline 0.0000 & 0.000 & 0.000 & 0.000 & 0.000 \\
0.0625 & 0.116 & 0.004 & 0.004 & 0.004 \\
0.1250 & 0.218 & 0.006 & 0.016 & 0.016 \\
0.1875 & 0.305 & 0.025 & 0.041 & 0.045 \\
0.2500 & 0.382 & 0.065 & 0.065 & 0.090 \\
0.3125 & 0.450 & 0.127 & 0.075 & 0.153 \\
0.3750 & 0.509 & 0.200 & 0.056 & 0.236 \\
0.4375 & 0.560 & 0.278 & 0.026 & 0.330 \\
\hline
\end{tabular}

Table 2. Signal error and restoration errors by the cumulant estimator $\rho^{(c)}$, after one, two, and three iterations, as a function of the effective depth $\epsilon z$.

\begin{tabular}{lllll}
\hline$\epsilon z$ & signal & iter $=1$ & iter $=2$ & iter $=3$ \\
\hline 0.0000 & 0.000 & 0.000 & 0.000 & 0.000 \\
0.0625 & 0.116 & 0.010 & 0.010 & 0.010 \\
0.1250 & 0.218 & 0.035 & 0.022 & 0.022 \\
0.1875 & 0.305 & 0.080 & 0.047 & 0.046 \\
0.2500 & 0.382 & 0.141 & 0.089 & 0.084 \\
0.3125 & 0.450 & 0.213 & 0.146 & 0.136 \\
0.3750 & 0.509 & 0.286 & 0.213 & 0.198 \\
0.4375 & 0.560 & 0.357 & 0.283 & 0.265 \\
\hline
\end{tabular}

grow again after the third iteration. This occurs because the initial estimate $f(\mathbf{r})$ is smaller than the exact density $\rho(\mathbf{r})$ (this is obvious from (2.1)(2.3)), so that at first the iterates underestimate the true solution. Because of the monotonicity property, the iterates always increase, so that (if the solution $\rho^{(m)}$ is larger than the true $\rho$, which is apparently the case here), they will start to overestimate the true density. The cumulant estimator $\rho^{(c)}$ underestimates the true density, and the values were stable within an accuracy of three digits after the third iteration. For comparison we give in column 2 of the tables the error before restoration, which is denoted

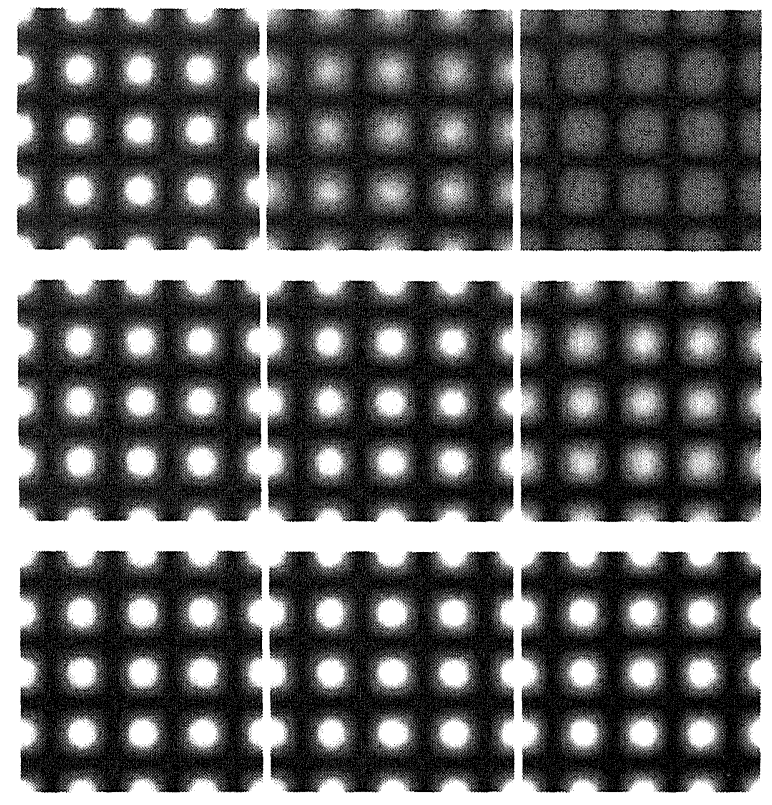

Fig. 2. Restoration of the trigonometric image: top row: attenuated test images; middle row, restoration by the cumulant estimator (iter $=2$ ); bottom row, restoration by the moment estimator (iter $=2$ ). In each row the first, fourth, and seventh layers are displayed from left to right. The original image in each layer is identical to the first image in the top row.

by "signal" and is computed according to (5.2) with $\widetilde{\rho}$ replaced by $f$.

Comparing the numbers in [5, table 4], we conclude that both the moment estimator with iter $=1,2$ and cumulant estimator with iter $\geq 2$ are more accurate than the layer method of [6], which gives a restoration error of 0.301 at the deepest layer. From the tables it is clear that the moment estimator, when run to convergence, overestimates the exact image densities. The first iterate, however, underestimates the exact values. Therefore, in the case of the moment estimator, we take the reconstruction corresponding to the intermediate value iter $=2$, which gives the best results.

In figure 2 we show the corresponding restored images. We rescale the restored values of the densities to make sure that they occupy the complete gray scale, which consists of the set of integer values from 0 to 255 . To ensure that a few outliers did not cause a large 
visual degradation of the resulting images, we constrained the approximate solutions $\widetilde{\rho}$ to lie between the known lower and upper bounds, i.e., $0 \leq \widetilde{\rho} \leq 1$. In each row, the first, fourth, and seventh layers are displayed from left to right, out of a total of eight depth layers. Since the exact density $\rho(\mathbf{r})$ does not depend on $z$, the original image in each layer is identical to the first image in row 1 . The top row contains the attenuated test images $f(\mathbf{r})$, the middle row contains the restoration by the cumulant estimator, and the bottom row contains the restoration by the moment estimator; both restorations are after two iterations. The images restored by the cumulant estimator are virtually identical to those of the layer method of Visser et al. [6]; see [5, figure 4]. Clearly, the largest improvement in restoration quality has been obtained by using the moment estimator. The reconstruction is not perfect, however: the central regions in the centers of the light circular regions are slightly overestimated. The calculations made here for the trigonometric image have been repeated for the "circle image" used in [5], leading to similar conclusions: the reconstruction errors are smallest when we use the moment estimator with iter = 2 , but the reconstructed images are still somewhat different from the original images; see figure 3. Nevertheless, a considerable improvement in restoration accuracy has been obtained by using the estimators developed here, which, in addition, are efficiently computable by using FFT methods.

\section{Summary}

In this paper we describe a refinement of the method developed in [5] for attenuation correction in fluorescence confocal microscopy by using FFT methods. Our approach, valid for weak attenuation, consists of multiplying the measured fluorescent intensity by a correction factor involving a convolution integral of the measured signal, which can be computed efficiently by an FFT-based algorithm. By a statistical reformulation of the problem we derive first-order moment and cumulant estima-
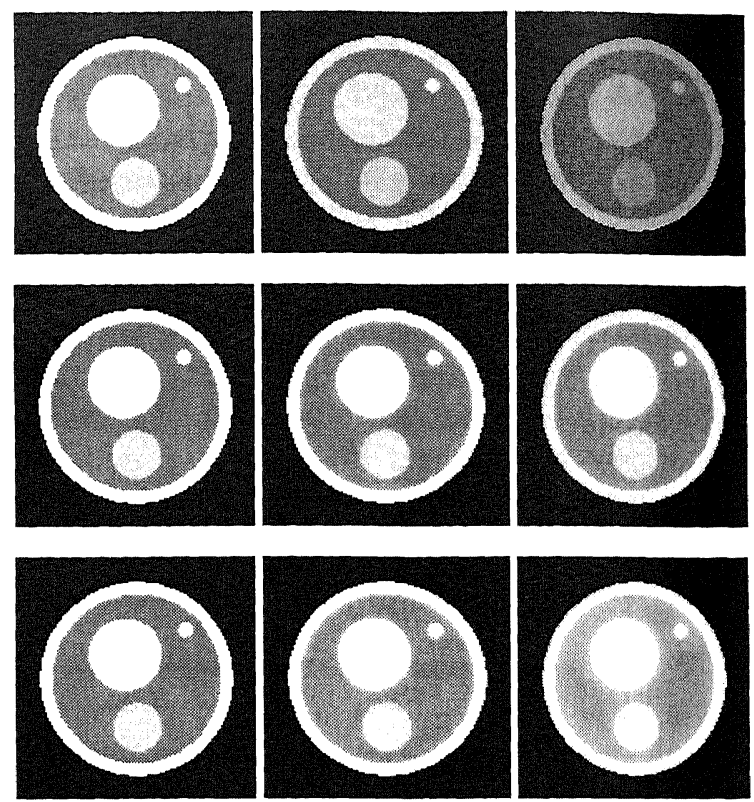

Fig. 3. Restoration of the circle image: Top row, attenuated test images: middle row, restoration by the cumulant estimator (iter $=2$ ); bottom row; restoration by the moment estimator (iter $=2$ ). In each row the first, fourth, and seventh layers are displayed from left to right. The original image in each layer is identical to the first image in the top row.

tors leading to a nonlinear integral equation for the unknown fluorescent density, which is solved by an iterative method. The algorithm is as follows:

1. Read the measured data $F_{i j k}, i=1, \ldots, N_{x}$, $j=1, \ldots, N_{y}, k=1, \ldots, N_{z}$.

2. Iteratively compute

$$
R_{i j k}^{(n)}=F_{i j k} G\left(\left(K * R^{(n-1)}\right)_{i j k}\right), \quad n=2,3, \ldots
$$

where $R_{i j k}^{(1)}=F_{i j k}$ and where $G(x)=(1-$ $\epsilon x)^{-1}$ and $G(x)=\exp (\epsilon x)$ for the moment and cumulant estimators, respectively.

In each iteration the convolution of the previous estimate is computed by means of the FFT (using the kernel $K$ of (2.11)). The first iterate of the new estimators coincides with the approximation used in [5] for very weak attenuation. It turns out that the moment estimator with 
two iterations gives the best results, which are more accurate than those of the layer method of [6]. Since only two iterations are needed, the advantage in computational efficiency over the layer method is retained. We conclude therefore that the combined results of [5] and this paper provide an efficient and accurate method for attenuation correction in confocal microscopy.

\section{References}

1. G.J. Brakenhoff, P. Blom, and P. Barends, "Confocal scanning light microscopy with high aperture immersion lenses," J. Microscopy, vol. 117, pp. 219-232, 1979.

2. E. Lukacs, Characteristic Functions, Griffin: London, 1960.

3. J.M. Ortega and W.C. Rheinboldt, Iterative Solution of Nonlinear Equations in Several Variables, Academic Press: New York, 1970.

4. W.H. Press, B.P. Flannery, S.A. Teukolsky, and W.T. Vetterling, Numerical Recipes, the Art of Scientific Computing, Cambridge University Press: New York, 1986.

5. J.B.T.M. Roerdink and M. Bakker, "An FFT-based method for attenuation correction in fluorescence confocal microscopy," J. Microscopy, vol. 169, pp. 3-14, 1993.

6. T.D. Visser, F.C.A. Groen, and G.J. Brakenhoff, "Absorption and scattering correction in fluorescence confocal microscopy," J. Microscopy, vol. 163, pp. 189-200, 1991.

7. T. Wilson and C.J.R. Sheppard, Theory and Practice of Scanning Optical Microscopy, Academic Press: New York, 1984.

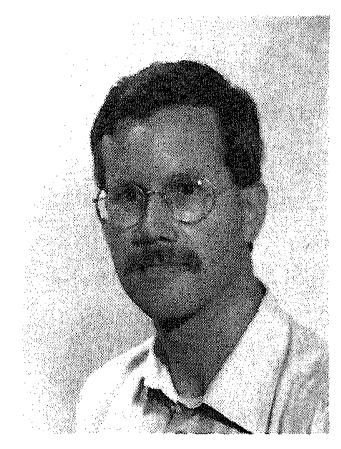

Jos B.T.M. Roerdink received his M.Sc. degree in theoretical physics from the University of Nijmegen, The Netherlands in 1979. After obtaining his Ph.D. degree from the University of Utrecht in 1983 and holding a two-year position as a Postdoctoral Fellow at the University of California, San Diego, both in the area of stochastic processes, he joined the Centre for Mathematics and Computer Science in Amsterdam. There he worked from 1986 to 1992 in image processing and reconstruction, in particular on magnetic resonance imaging, confocal microscopy, and mathematical morphology. He is currently associate professor of computing science at the University of Groningen, the Netherlands. His research interests include group-theoretical methods in mathematical morphology, multiresolution representations, computer vision, and graphics. 